\title{
Pre-empting understanding problems in L1/L2 conversations: Evidence of effectiveness from simulated emergency calls
}

\author{
Abstract \\ This study presents a quantitative analysis of pre-emptive strategies used by first language \\ speakers in talk addressed to second language speakers with moderate proficiency. The strategies \\ identified in the conversations are reformulation, simplification, left-dislocation, and \\ decomposition. The data come from simulated emergency calls in which an (L1) 'operator' \\ instructed a (L2) 'caller' how to put a 'patient' (a manikin) in the recovery position. The \\ conversations were coded for how many (and which) strategies the operators used and for \\ whether or not the instruction was understood by the caller. The analysis showed that there is a \\ relationship between the average number of strategies used and the chance of successful \\ understanding; a greater number of strategies employed led to fewer understanding problems. \\ The study shows that in this context of instruction, L1 speakers orient to potential problems of \\ understanding for L2 addressees by using recognizable pre-emptive strategies, and that these \\ strategies are effective in reducing the likelihood of understanding problems occurring.
}

\section{Introduction}

With increasing globalization, more and more institutional interactions involve users who are second language (L2) speakers. This may create challenges in establishing intersubjectivity between the parties, especially when the proficiency level of the L2 speakers is low. In medical emergencies, misunderstandings or lack of understanding may have serious consequences for 
both safety and efficiency (Grow et al. 2008). For instance, fatal incidents have occurred as a direct result of miscommunication between emergency call operators and callers with minority backgrounds (Svennevig 2012). A recent literature review on the effects of language barriers on the quality of prehospital care showed that L2 callers experienced delayed, less accurate dispatch and operators were less able to provide medical direction (Tate 2015).

While what constitutes efficient and effective communication in an L2 emergency telephone setting is not well researched (Meischke et al. 2010), some recent studies have made concrete contributions to identifying specific problems associated with such emergency calls. For instance, Larsen (2010) showed that L2 speakers were more likely to use a statement of need ("I need the paramedics please?") when presenting their request for assistance. This format displays a lower degree of entitlement to service than explicit requests and are more easily rejected by the call-taker. A case study by Osvaldsson, Persson-Thunquist \& Cromdal (2013) describes how the operator makes efforts to pre-empt understanding problems by actively soliciting a clear display of understanding and by designing sequential environments for so-called en passant verification of comprehension. A quantitative analysis of simulated emergency calls by Gerwing \& Indseth (2016) found the same tendency; more than half of the operator's responses to informative turns by the L2 callers were explicit displays of understanding presented for the caller to confirm or correct. By contrast, Svennevig's (2012) analysis of a fatal emergency call showed how the operator's failure to display understanding had damaging consequences in that undetected misunderstandings engendered misalignment and hostility.

The current study analyses L1 speakers' instructions to L2 callers, specifically how emergency call operators anticipate and attempt to prevent problems of understanding. Although providing medical directions on the phone can be both critical for patient safety and particularly 
challenging with L2 callers, little research has elucidated the topic. Painter et al. (2014) asked both L2 speakers and emergency operators to identify the phrases or words in CPR instructions that might be challenging and to propose alternatives to help understanding; however, the resulting, simplified English instructions were tested using simulated calls with native English callers and not L2 callers. We have found only one study that focused on instructions for L2 callers: Meischke et al. (2015) found that allowing an operator to rephrase CPR instructions when the callers did not understand (vs. repeating them rigidly) led to only slight, non-significant improvements in how callers carried out the instructions. However, the single operator in the experiment was given "alternative, simple ways to rephrase and elaborate on the 9-1-1 script in the case of misunderstandings during the call interactions" (p. 1050), but neither the alternative phrases were provided nor what the operator actually did were reported. Thus it is unclear what impact, if any, these alternative phrases or behaviours had on caller understanding.

Besides a need to generate concrete, practice-relevant solutions in this specific context, basic research on language use also benefits from advances in understanding the challenges to intersubjectivity involved in L1/L2 interaction and how these challenges can be remedied. Our approach to this question is studying how L1 speakers spontaneously and intuitively use preemptive strategies in order to prevent understanding problems from arising when speaking to L2 speakers with moderate proficiency.

Speakers in general seem to orient to a preference for preventing conversational problems from arising over repairing them after they have arisen (Clark 1994), much in line with the dictum formulated by Desiderius Erasmus (1466-1536) that "Prevention is better than cure." Pre-emptive strategies are understood as any conversational practice that reduces the progressivity of the talk in the service of explaining or contextualizing a part of the utterance-in- 
progress, or otherwise making it more salient or accessible (Svennevig 2010). An example can be reformulating or paraphrasing something one has just said in simpler or more explicit terms. Here is an example:

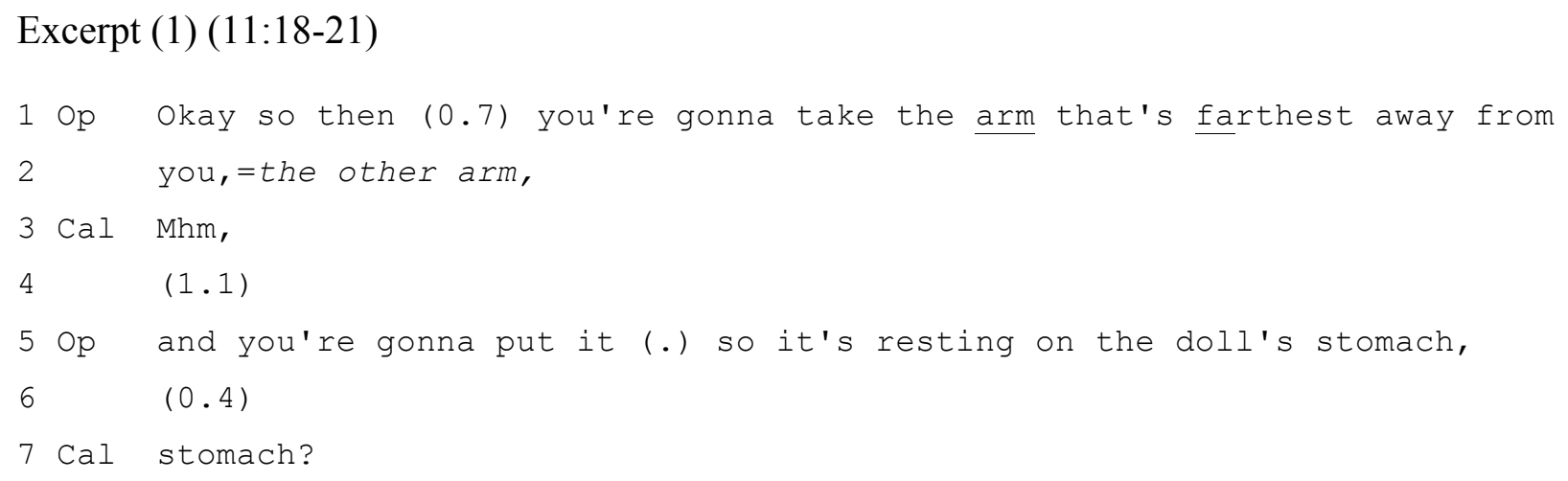

In this example, the operator produces a potentially complete turn constructional unit in line 1, but immediately initiates a self-repair by rephrasing one of the expressions used ("the arm that's farthest from you") in a transition space repair ("the other arm") (Schegloff, Jefferson and Sacks 1977). This self-repair halts the progressivity of the talk by extending the turn and thus delaying the response of the interlocutor. The material added is a reformulation of one of the referring expressions, providing a potentially simpler expression and a different way of identifying the referent. This addition may thus be understood as promoting understanding (referent identification) over progressivity (advancing the activity of giving instructions), and thus qualifies as a pre-emptive strategy. Such practices are thus forward-oriented, in the sense that they are not responsive to an indication of trouble by the interlocutor (for instance a repair initiation) but are oriented towards preventing a potential problem of understanding from arising in the following talk. 
In this study, we analyze the frequency of the use of pre-emptive practices in simulated emergency calls involving L1 operators instructing L2 callers on a first aid task (how to put someone into the "recovery position"). We assess whether or not the operators' pre-emptive practices result in fewer manifestations of understanding problems. The research questions we aim to answer are: First, which pre-emptive practices are used by L1 speakers when instructing L2 speakers of moderate proficiency (if any)? Second, does the use of pre-emptive strategies lead to fewer understanding problems for the L2 speakers?

\section{Pre-emptive practices in conversation}

Although there has been a substantial amount of research into the language usage and communicative competence of L2 speakers, less research has been devoted to how L1 speakers modify their linguistic and interactional practices to accommodate to the linguistic resources of L2 interlocutors. But some studies have addressed this topic, in both educational and noneducational settings.

The history of this research topic dates back to Ferguson (1975), who coined the term foreigner talk to describe how first language speakers modify their talk in order to accommodate to the (perceived) proficiency level of second language speakers. In the following years, several researchers within the field of second language acquisition continued this line of research and described a broad range of grammatical and interactional adjustments made by L1 speakers (e.g. Hatch 1978, Long 1996, Varonis and Gass 1985, Pica, Young and Doughty 1987). Much of this research has been criticized by researchers in the field of Conversation Analysis (CA) for being focused only on one party to the interaction, the L1 speaker, and for not analyzing the coconstruction of meaning by both parties (Firth \& Wagner 1997). In recent years, the study of 
L1/L2 interaction has increasingly adopted a conversation analytic methodology, and the review of previous research on preemptive practices will thus mainly focus on these studies.

Several CA researchers have described recurrent practices of self-repair oriented to preempting potential understanding problems, especially concerning potentially problematic referring expressions. For instance, Mazeland (2007) showed how parenthetical sequences may be inserted into an ongoing turn in order to clarify, specify, or exemplify a referring expression, thereby enhancing the comprehensibility of it. Heritage (2007) described how the transition space following a turn constructional unit might be used to add information about a referent or to check the recognizability of it. And Svennevig (2010) further described how turn constructional units containing a potentially problematic referring expression might be expanded in the course of their production in order to accommodate identification-relevant information.

A range of studies have analyzed questions addressed to $\mathrm{L} 2$ speakers that are immediately reformulated in other words, usually by offering candidate answers to the original question (Gardner 2004, Kasper and Ross 2007, Svennevig 2013). This practice is described as a way of pre-empting problems in understanding of the question by presenting models of relevant answers to it. Another practice associated with talk addressed to L2 speakers is decomposing complex information into smaller units and delivering them one at a time (in installments), allowing the interlocutors to display their understanding after each part (Svennevig 2015).

Common to these studies is that they are qualitative descriptions of the interactional practices themselves. They do not attempt to assess whether the practices are helpful or not, that is, whether or not they result in enhanced mutual understanding between the parties. To our knowledge, no studies to date have tried to assess whether pre-emptive practices in conversation are actually effective. This paper attempts to do just that. 


\section{Data collection ${ }^{1}$}

\section{Participants}

Seventeen dyads participated. Each dyad consisted of one moderate L2 English speaker (the “caller”) and one fluent L1 English speaker (the "operator”). The 17 L2 callers were recruited from 400-500 level English courses at the English Language Centre of a Canadian university and received a gift card for participating. During the recruitment process, L2 students who were interested in participating were given copies of the consent and video release forms, which were written in English. Based on the classes from which they had been recruited, these callers (10 female, 7 male) could be considered moderately proficient English speakers and were, on average, 24.94 years old $(S D=5.84, M I N=18, M A X=42)$. They reported the following as their native languages: Spanish (5), Japanese (3), Portuguese (3), Korean (3), Arabic (1), Chinese² (1), and Mandarin (1). The 17 L1 operators were all Psychology students from the same university and were recruited using the online Psychology participant pool, receiving course credit in return for their participation. The operators (10 female, 7 male) were all fluent English speakers; 15 reported having English as their native language and two reported a different native language. They were, on average, 21.53 years old $(S D=3.87, M I N=18, M A X=33)$.

\section{Procedure}

Each participant arrived at the Human Interaction Lab and was met by the experimenter (the second author). They read and signed consent forms. After introducing the participants and informing the operator (the psychology student) that the other participant (the caller) was a

\footnotetext{
${ }^{1}$ This study received ethical approval from the University of Victoria Human Research Ethics Board (Ethics protocol \# 12-399) and the Elon University Institutional Review Board (Protocol \# 13-056).

${ }^{2}$ One participant did not specify the variety of Chinese.
} 
student at the English Language Centre, the experimenter asked the caller to move to an adjacent office to do an unrelated task for a few minutes. The experimenter then gave the operator written instructions for how to put a small wooden manikin into the recovery position (see Figure 1) and gave the operator time to practice on the doll. There were five steps of instructions, see Table 1.

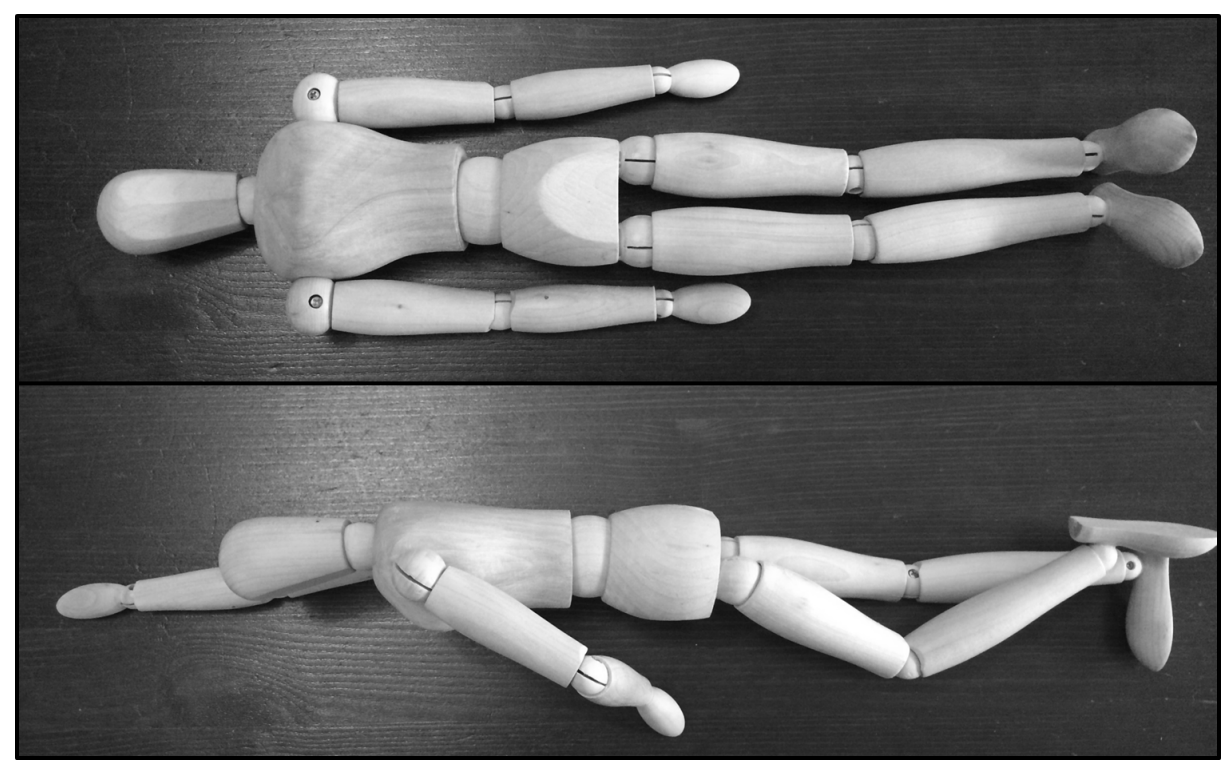

Figure 1. The wooden manikin in the neutral position (top) and in the recovery position (bottom)

Table 1. Five steps of written instructions provided to operators

1 Position the arm closest to you so that the arm is above the doll's head

2 Position the arm furthest from you so it is resting on the doll's stomach

3 Bend the far knee a little bit and put the foot flat on the table

4 Put one of your hands on the doll's shoulder and one on the bent knee

5 Carefully roll the doll towards you so it is positioned on its side 
After a few minutes, the experimenter checked that the operator could carry out the instructions correctly, returned the doll to a neutral position, and invited the caller to return to the room. The experimenter then told the two participants that they were to imagine that the caller's friend (represented by the doll) had "passed out" and was unresponsive (taking time to explain "passed out" to the L2 caller implicitly informed the operator about the caller's English proficiency). The caller was to imagine that he or she had already called the emergency line (9-11) and explained the situation to the operator (the other participant), who would now instruct the caller on how to put the friend into a safe position while waiting for the ambulance. The experimenter then placed a wide partition between the two participants so they could not see each other but explained that they could talk freely (see Figure 2 for an overhead sketch of the seating arrangement, and Figure 3 for a screen shot of the camera view).

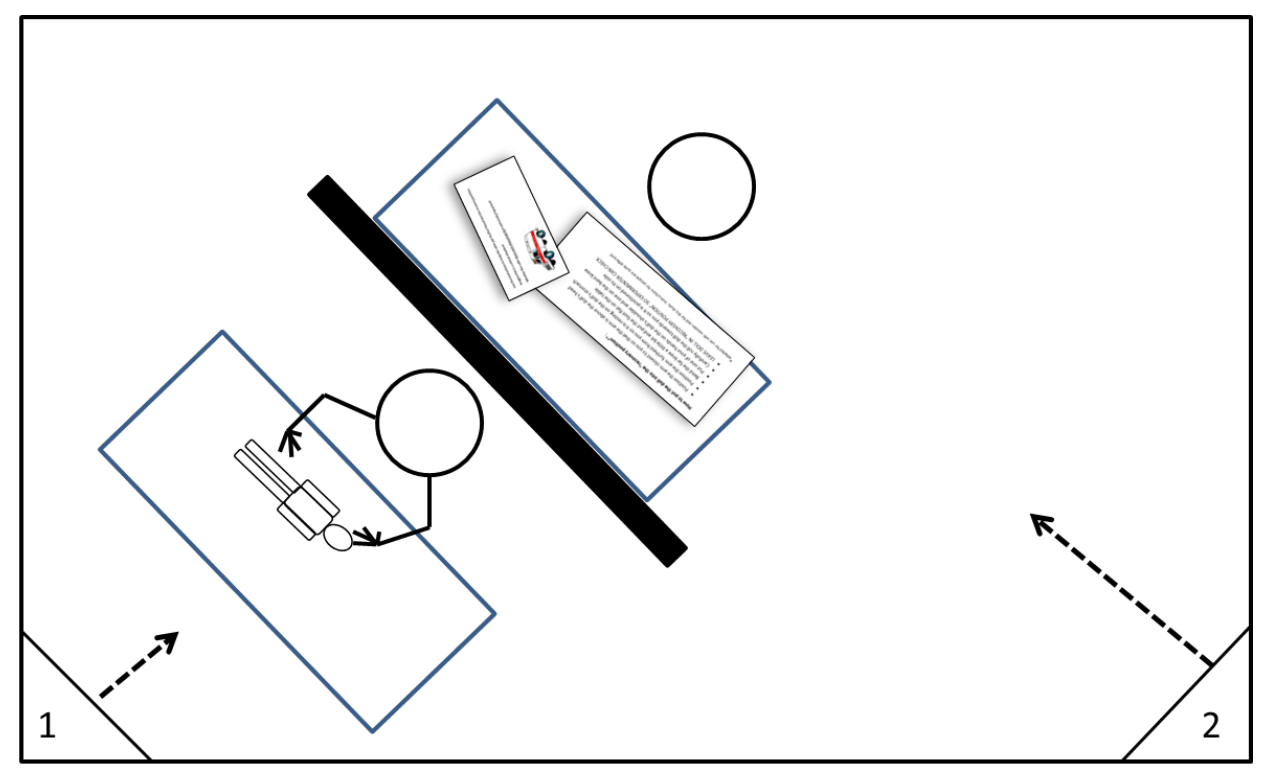

Figure 2. Overhead sketch of lab set up. Note that participants were separated by a partition: camera 1 captured a front view of the ESL caller and manikin; camera 2 captured a side view of the operator. 


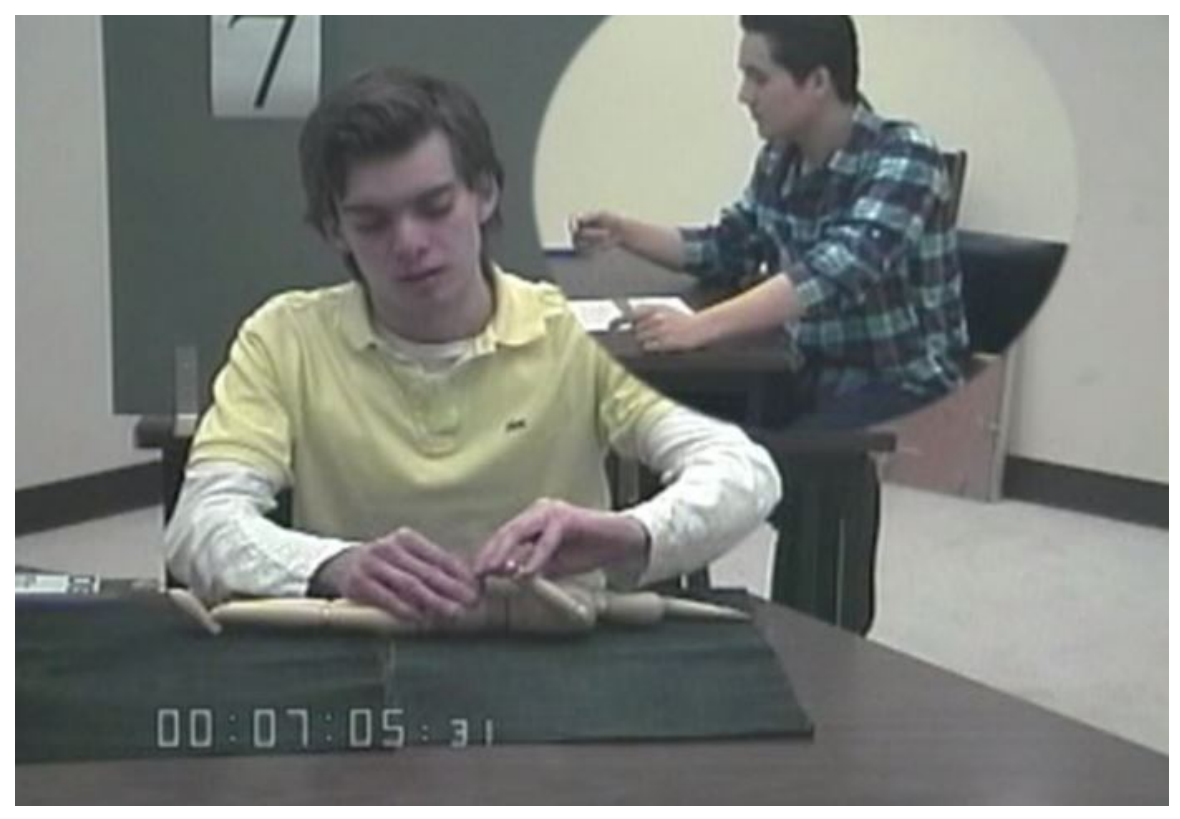

Figure 3. Split-screen camera view of participants: front view of the caller and manikin, side view of the operator (in inset circle), who is seated behind the partition.

After answering any final questions, the experimenter left the room, and the participants did the task. When they were finished, the experimenter gave them questionnaires about their impression of the task and their dialogue (data not presented here) and a short form that included a question about their experience with first aid generally and the recovery position specifically. None of the L2 callers reported having experience or familiarity with the recovery position; eleven of the L1 operators were familiar with it. When the experiment was over, participants watched the video of their dialogue and had the opportunity to discuss the task, their experience, and the overall purpose of the study. Finally, they read and signed video release forms stating the purposes for which they gave us permission to use their video (e.g., for analysis only, for photos in journal articles, for conference presentation examples). 


\section{Transcription}

Using the annotation software ELAN (Wittenberg et al., 2006), a research assistant transcribed the participants' speech. All pauses were generated automatically by the annotation program. These annotations were exported into Word documents to create transcripts that were used in conjunction with the annotated video. All utterances were numbered. An utterance was considered to be as long as the participant spoke until the other person responded or there was a pause.

\section{Analytic procedure}

The analysis is based on a combination of Conversation Analysis (CA) (Sidnell and Stivers 2013) and Microanalysis of Face-to-face Dialogue (MFD) (Bavelas, Gerwing, Healing, and Tomori, in press). In accordance with CA, the pre-emptive strategies were analyzed in their sequential context, and the interpretation of the individual turn at talk was based on the recipient's display of understanding in the next turn at talk (next turn proof procedure, Sacks, Schegloff and Jefferson 1974). The experimental design and the quantitative analysis were derived from the tradition of MFD. A potential incompatibility between the traditions concerns CA's principle of using naturally occurring talk (Sacks 1984). However, lately CA studies have increasingly adopted experimental designs using laboratory data, and we agree with Kendrick (2017) that CA has reached a stage where such research may constitute a valuable addition to naturalistic observation. And in our case, using naturalistic data would not allow us to achieve the aim of comparing different conversations and quantifying the results, since real world emergency calls differ from each other in too many respects. We thus consider this combination of methodologies an apt solution to the goal of addressing a research topic that neither of the methodologies alone would allow us. 


\section{Pre-emptive windows}

For each step of the instructions, pre-emptive strategies could be employed in a window starting from the first utterance pertaining to that step of instructions. The end of the window was either when the caller exhibited a sign of trouble (e.g., with a repair initiation, after which any operator adjustments could no longer be considered pre-emptive) or, if the caller displayed no sign of trouble, when the operator moved on to the next step of instructions. Decisions about the beginning and end of the pre-emptive window for each step of instructions were made by consensus between the first and second authors.

\section{Identification of pre-emptive strategies}

As noted above, we consider pre-emptive strategies as turn-constructional practices that reduce the progressivity of an utterance-in-progress in the interest of making it more comprehensible or contextually relevant. We started looking for such practices by considering any modification of the written instruction that seemed to enhance the comprehensibility of the instruction. By an inductive process of examining the conversations in an in-depth, qualitative interaction analysis, the first author made a collection of 69 instruction sequences that included some form of preemptive strategy. Excluded were modifications that did not seem oriented to enhancing understanding. One case in point is repetition. Just repeating an utterance or a part of it is not in itself oriented to enhancing understanding. It may indeed be oriented to enhancing the perception of the utterance, but we were specifically interested in pre-emptive practices dealing with potential problems of understanding. Further analysis of the collection of excerpts yielded that the strategies could be grouped in four more specific types. They were lexical simplification, 
reformulation, left-dislocation of new referents and decomposition. These are presented in more detail below.

Lexical simplification is using a simpler word or expression than was used in the written instructions. Here is an example, where the verb "position" in the written instruction (provided in square brackets) is substituted by the simpler verb "rest" in the oral instruction:

\section{Excerpt (2) (15: 80-81)}

[Step five: Carefully roll the doll towards you so it is positioned on its side]

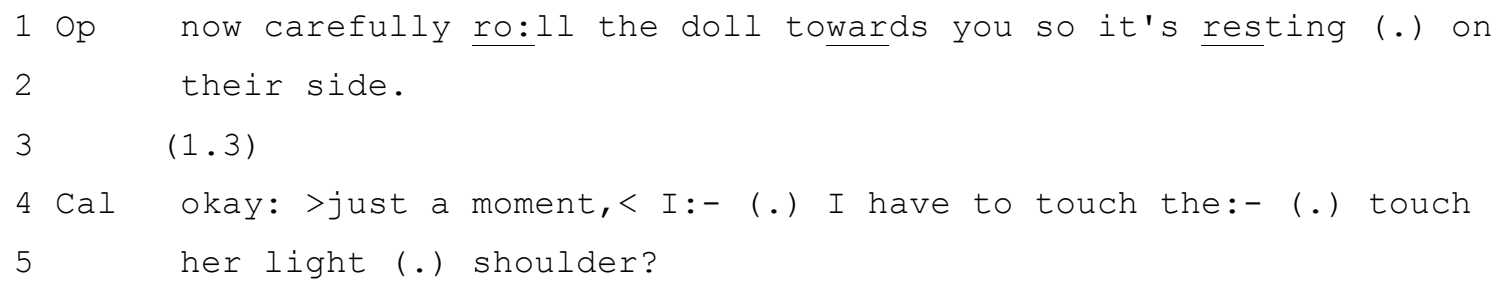

We did not include lexical modifications that were just stylistic variations and did not contribute to simplifying the instruction, such as referring to the doll as "the person" or "your friend", or changing from the singular pronoun it to the plural they (their) as in lines 1-2 above.

Reformulation is a form of self-repair in which the speaker after the completion of a turn constructional unit or a constituent part of it produces a second version of it in a simpler or more contextually adapted manner. This may involve either giving two different descriptions of a single referent or reformulating a whole clause. Here is an instance that includes both types:

\section{Excerpt (3) (8: 15-20)}

[Step one: Position the arm closest to you so that the arm is above the doll's head]

1 Op Okay uhm so the f:irst step to put them: (0.7) into thee: s:afe 


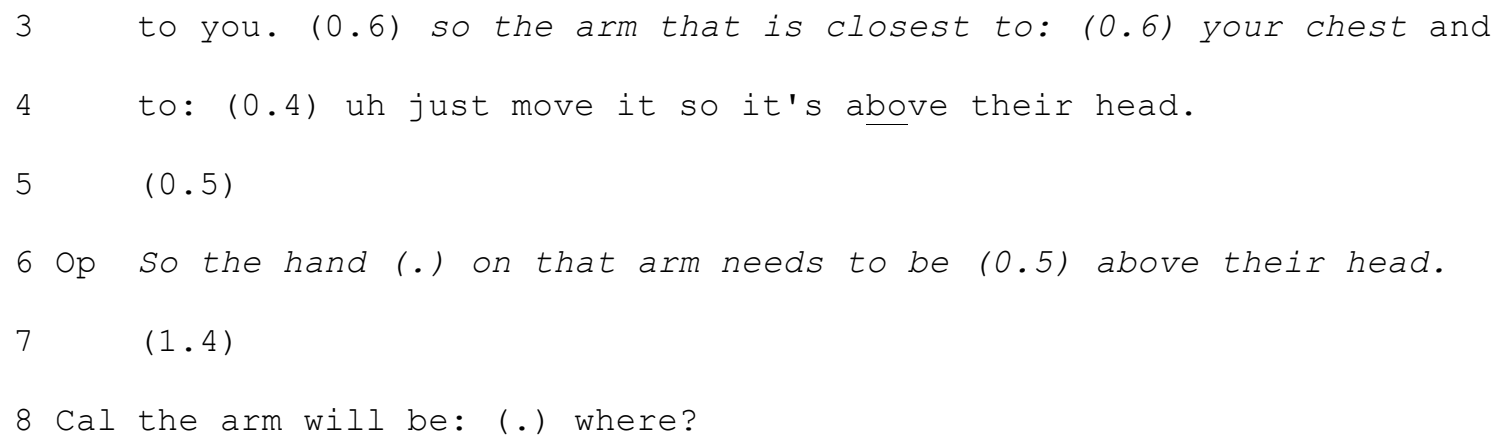

First there is a reformulation in which the referring expression "the arm that is closest to you" is made more precise by being repeated with additional identifying information ("closest to your chest"). Second, there is a reformulation of a full clause in line 6 , where the gist of the whole instruction is repeated in a modified and condensed form. Both reformulations are introduced by 'so', which has been described as a discourse marker introducing a conclusion or an inference from previous talk (Schiffrin 1987). ${ }^{3}$ Reformulations may occur inside a complex TCU, such as the first example, or in the transition space following a TCU, as in the second case.

Sometimes there are several reformulations in a single instruction. Here is an example where the operator repeatedly tries to find other ways of explaining the instruction step:

\section{Excerpt (4) (01:74-81)}

[Step five: Carefully roll the doll towards you so it is positioned on its side]

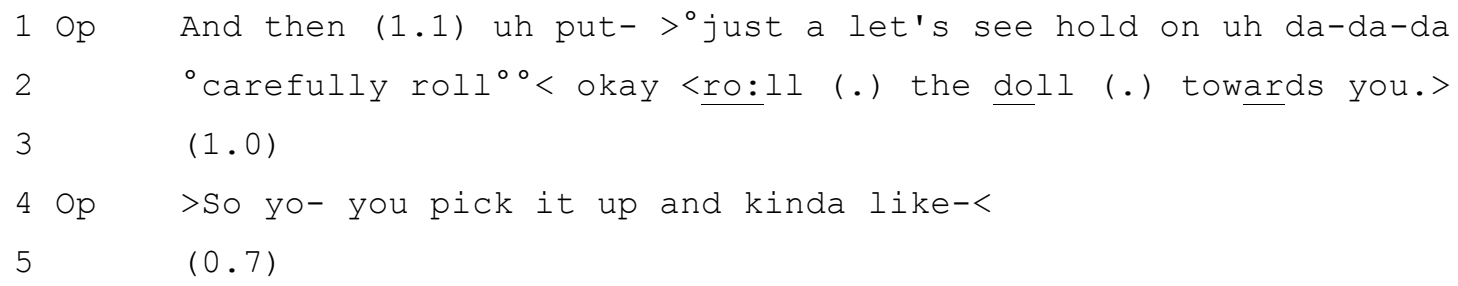

${ }^{3}$ Reformulations are not all introduced by this discourse marker, though. And the marker may certainly be used with other functions than introducing reformulations, such as the occurence found in line 1. 


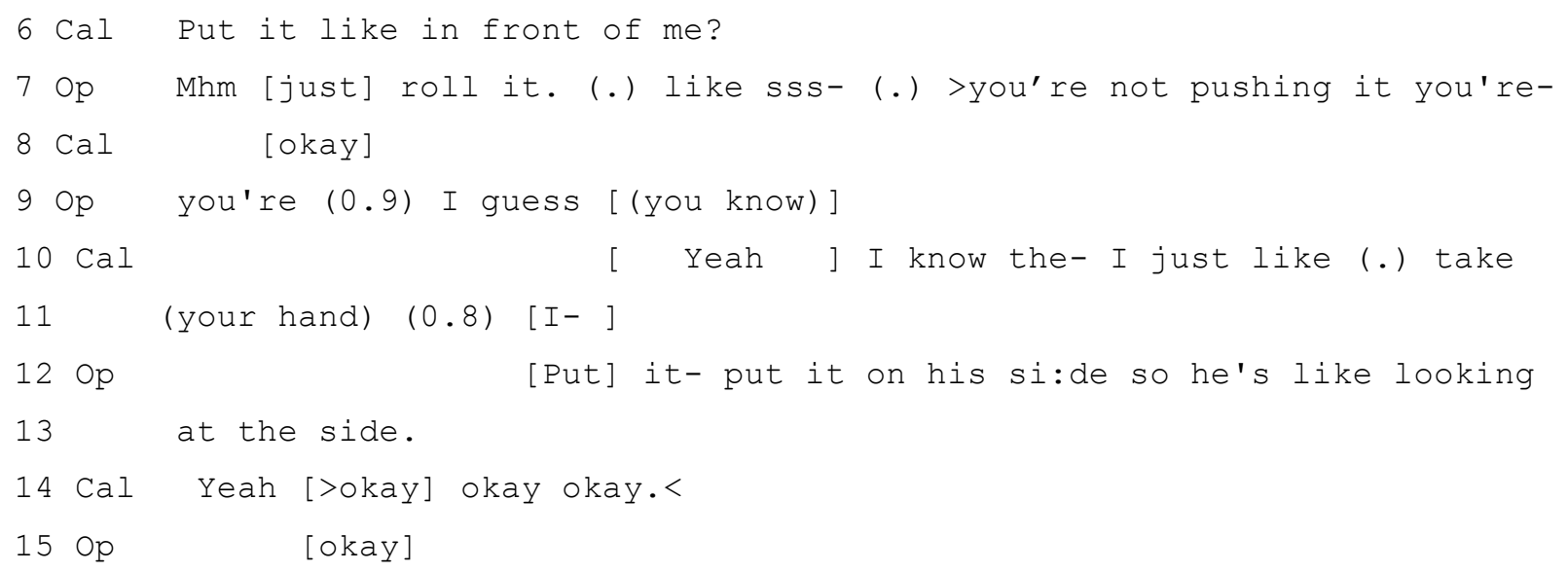

First in line 4 the operator produces a reformulation in a transition space repair, adding the information that the caller should "pick it up". He aborts the utterance mid-course, and the caller attempts to complete it in line 6 . By formulating what he thinks the operator is trying to say, his collaborative completion constitutes an understanding check. The completion is partially accepted in that the operator confirms but then recompletes the utterance in a different way ("just roll it"). A new attempt at reformulating the instruction is produced in line 7 in a new transition space repair. Here, the operator seems to be aiming at an illustration of the action ("like sss-") but this is also aborted and instead replaced by a reformulation using an antonymic expression ("not push"). Finally, in line 12, there is yet another reformulation, this time using a synonymous expression with the simple, high-frequency verb 'put' ("put it on his side”). A problem for coding reformulations may be what to do with aborted utterances. In this excerpt, one of the potential reformulations ("like sss-" in line 7) did not provide any alternative formulation and was thus not counted. The other ones that were also aborted (lines 4 and 7) did in fact provide an alternative formulation and were thus counted. So this instruction was coded as containing three reformulations. 
Left-dislocation is a strategy of first presenting a referring expression as a free-standing fragment outside the sentence frame and then initiating a sentence where the expression is used. Here is an example:

Excerpt (5) (16:11-12)

[Step two: Position the arm furthest from you so it is resting on the doll's stomach]

1 Op so now the other a:rm (0.7) so the one furthest from you, (0.4)

2 you're gonna take that arm, (0.5) and you're gonna place the ha:nd on the doll's stomach.

$3 \quad(0.9)$

4 Cal doll's stomach.

Here the referent (the body part) is first introduced without being integrated in a sentence ("the other arm so the one furthest from you"). And only afterwards the sentence starts and the reference to the arm is done again, this time integrated into the sentence frame ("that arm"). This construction is frequently used to introduce new referents into discourse (Lambrecht 1994). It may enhance the comprehensibility of an utterance by making the new referent more salient and by separating the two actions of reference and predication.

Decomposition is a strategy by which information is delivered in several small chunks installments - instead of in one complex utterance (Clark 1996, Svennevig 2015). Each installment (but the last) is delivered with rising intonation, projecting that there is more to come. After each installment there is a pause, and frequently, the addressee produces an acknowledgement token during this silence. Here is an example:

Excerpt (6) (11:1-5) 
[Step one: Position the arm closest to you so that the arm is above the doll's head]

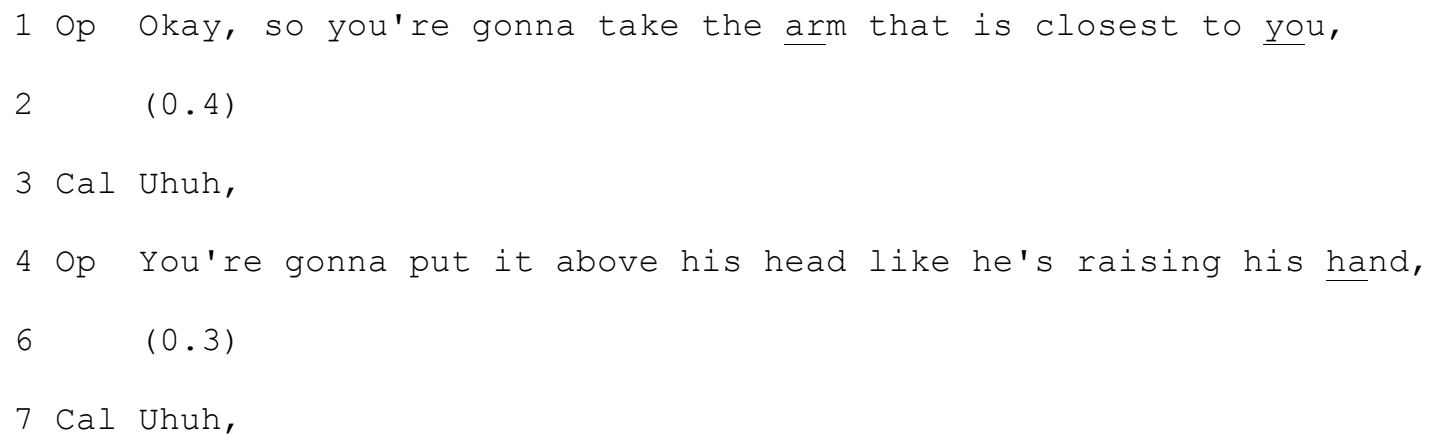

Here the instruction is divided up into two parts, first a clause identifying the body part, and second a new clause providing instructions about what to do with it. Just like left-dislocation, this practice enhances comprehensibility by presenting smaller pieces of information at a time. The difference between them is that the first is a purely syntactical separation of the two pieces of information, whereas the second is an interactional practice involving temporal and turnconstructional separation of the two pieces.

Many instructions involve the use of several pre-emptive strategies at once. We saw above that several reformulations could occur in a single instruction (excerpts 3 and 4). But there can also be several different strategies present in an instruction. Let us take the first extract above (1) as an example, repeated here:

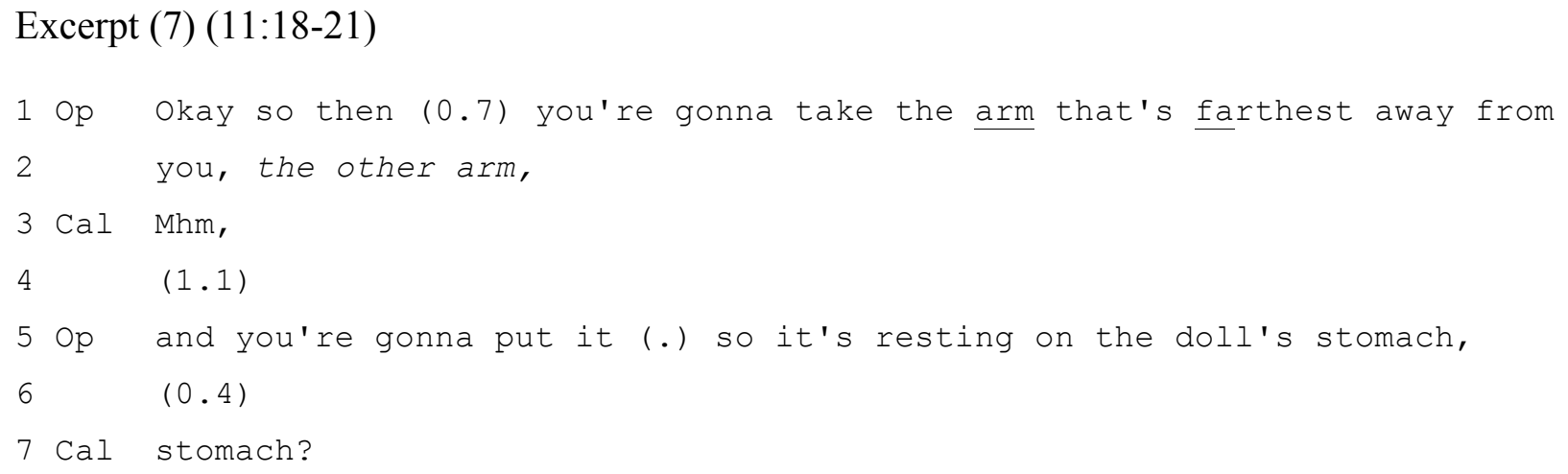


Here we have several strategies present. First, there is a reformulation of the body part ("the other arm"), second, there is decomposition (dividing the instruction up into two parts with a pause between the parts) and third, there is simplification (put for position). So in the process of coding, this instruction has been assigned three pre-emptive strategies.

\section{Analysis of pre-emptive strategies}

The first author formulated operational definitions of the strategies and used them as a basis for making analytical decisions about all operator utterances within pre-emptive windows. In order to determine whether the operator used any pre-emptive strategies, each step in the oral instruction was compared to the written instruction. Sometimes operators' oral instruction steps did not include any strategies at all, whereas many of them included several (as illustrated in Excerpt (7), above). Above we have seen that excerpt (3) involved two reformulation strategies in the same instruction. Excerpt (5) is presented as an example of left-dislocation, but it also includes a reformulation ("the other arm so the one furthest from you"). The highest number of strategies used in a single instruction step was five.

When this analysis was finished, the second author re-organized the excerpts by step (i.e., all step one pre-emptive windows in one file, all step two in another, and so on), affording an additional layer of systematicity in decision making. Using the operational definitions and the reorganized excerpts, the second author reviewed all decisions and flagged any disagreements, which the two analysts then resolved by consensus to create the final data set of each preemptive strategy. 


\section{Reliability check}

To conduct an inter-analyst reliability test, we created a random selection of 23 utterances (approximately 20\%) from the 123 utterances that had been included in the analysis. After training, the fourth author used the operational definitions to analyze the 23 utterances independently. When her results were compared to the results obtained by the first and second authors, reliability was $93 \%(107 / 115)$ based on giving credit for each of five decisions that analysts had to make for each utterance analyzed (i.e., whether the utterance included a reformulation $\mathrm{Y} / \mathrm{N}$, left dislocation $\mathrm{Y} / \mathrm{N}$, lexical simplification $\mathrm{Y} / \mathrm{N}$, decomposition $\mathrm{Y} / \mathrm{N}$, or no strategy $\mathrm{Y} / \mathrm{N})$.

\section{Analysis of success}

To analyze whether the pre-emptive strategies were successful, we examined both caller utterances and caller actions at the end of each pre-emptive window. Caller displays of understanding could be implicit (e.g., saying "m-hm" or "OK") or explicit (e.g., repeating or rephrasing the instruction with prosody that indicated understanding). Caller utterances that indicated non-understanding could be explicit indications of non-understanding ("I don't understand") or repair initiations targeting semantic aspects of the previous utterance, such as reference assignment, identification of action type etc. For instance, in one example (extract (3) below) the caller initiates repair by saying: "the arm will be: (.) where?", thus displaying a problem of identifying a referent in the previous talk (the location of the arm).

Using video also allowed us to observe the callers' manipulation of the doll, thus showing whether or not the instructions were carried out correctly. Caller actions that demonstrated misunderstanding (i.e., errors) were defined as a manipulation of the doll that was incorrect, when compared to the oral instructions. To be considered an error, the manipulation 
must have appeared purposeful with a clear trajectory and aim. Examples of errors were: the caller mistakenly moving a leg after being instructed to move the arm; moving the whole doll closer to the edge of the table instead of moving the arm that is closer; or crossing the doll's arms instead of moving one above the head. Instances that were not coded as error were cases where the callers touched and fidgeted with the doll without actually moving it very much. Caller errors were identified by a research assistant working independently with no information about the purpose of this study.

Combining caller utterances and actions, the criteria for lack of success at the close of each pre-emptive window was either a verbal display of non-understanding or an error when carrying out the instruction. The criteria for success at the close of each pre-emptive window required both a caller display of understanding (implicit or explicit) and a correct action. In other words, lack of success could be a caller utterance that displayed non-understanding to the operator (whether the caller had manipulated the doll yet or not), or it could be an incorrect action that was invisible to the operator, but observable to analysts viewing the video.

\section{Results}

With 17 pairs of participants and 5 steps of instruction, the total number of realized instructions is potentially 85 . However, in four cases the operators skipped a step in the instruction (step 4), and so the actual number of instructions realized was 81 .

A first observation that emerged from the analyses was that the preemptive strategies were commonly used. As can be seen in Table 2, there were a total of 129 strategies manifested in the 81 instruction steps altogether. The strategy that was used the most was decomposition (56 instances) followed by reformulation (39) and simplification (30). Left-dislocation was used less often (4). 
Table 2: Number of instances of pre-emptive strategies in the data

\begin{tabular}{|l|c|}
\hline Strategy & Instances \\
\hline Decomposition & 56 \\
\hline Left-dislocation & 4 \\
\hline Reformulation & 39 \\
\hline Simplification & 30 \\
\hline Total & 129 \\
\hline
\end{tabular}

If we look at the individual profiles of the participants, decomposition was also the strategy that was used the most, in the sense that it was used by all participants $(n=17)$, whereas simplification was used by 15 and reformulation by 14 of the participants. We can also note that most participants $(n=10)$ used one or more strategy in every step of instruction.

To see how these strategies correlated with the success of the instruction, we may start by looking at the numbers for all instruction steps taken together. As can be seen in Table 3, understanding problems and misunderstandings were quite common in the conversations, being manifested in 57 of the 81 instructions. ${ }^{4}$ Another general pattern is that most instructions were in fact presented with one or more pre-emptive strategies. Only 12 of 81 instructions did not include any of the pre-emptive strategies. Finally, we can see that the success rate increased with the number of strategies used. This is visualized in Figure 4. None of the instructions that were formulated without pre-emptive strategies were successful. The use of one strategy raised the success rate to $18 \%$, two strategies had a success rate of $40 \%$ and three or more strategies raised the rate to $60 \%$.

\footnotetext{
${ }^{4}$ The outcomes have here been termed 'success/failure' for brevity's sake, but this does not mean that the instructions failed to be carried out in these cases. Often, a repair sequence would re-establish mutual understanding and the instruction would be carried out successfully. 'Failure' thus simply indicates either a display of non-understanding or that the instruction was not carried out as it was formulated in the first attempt.
} 
Table 3: The relation between number of strategies and outcome of the instruction

\begin{tabular}{|l|l|l|l|}
\hline $\begin{array}{l}\text { Number of } \\
\text { strategies }\end{array}$ & Failure & Success & Total \\
\hline 0 & $12(100 \%)$ & $0(0 \%)$ & 12 \\
\hline 1 & $28(82 \%)$ & $6(18 \%)$ & 34 \\
\hline 2 & $9(60 \%)$ & $6(40 \%)$ & 15 \\
\hline $3-5$ & $8(40 \%)$ & $12(60 \%)$ & 20 \\
\hline Total & $57(70 \%)$ & $24(30 \%)$ & 81 \\
\hline
\end{tabular}

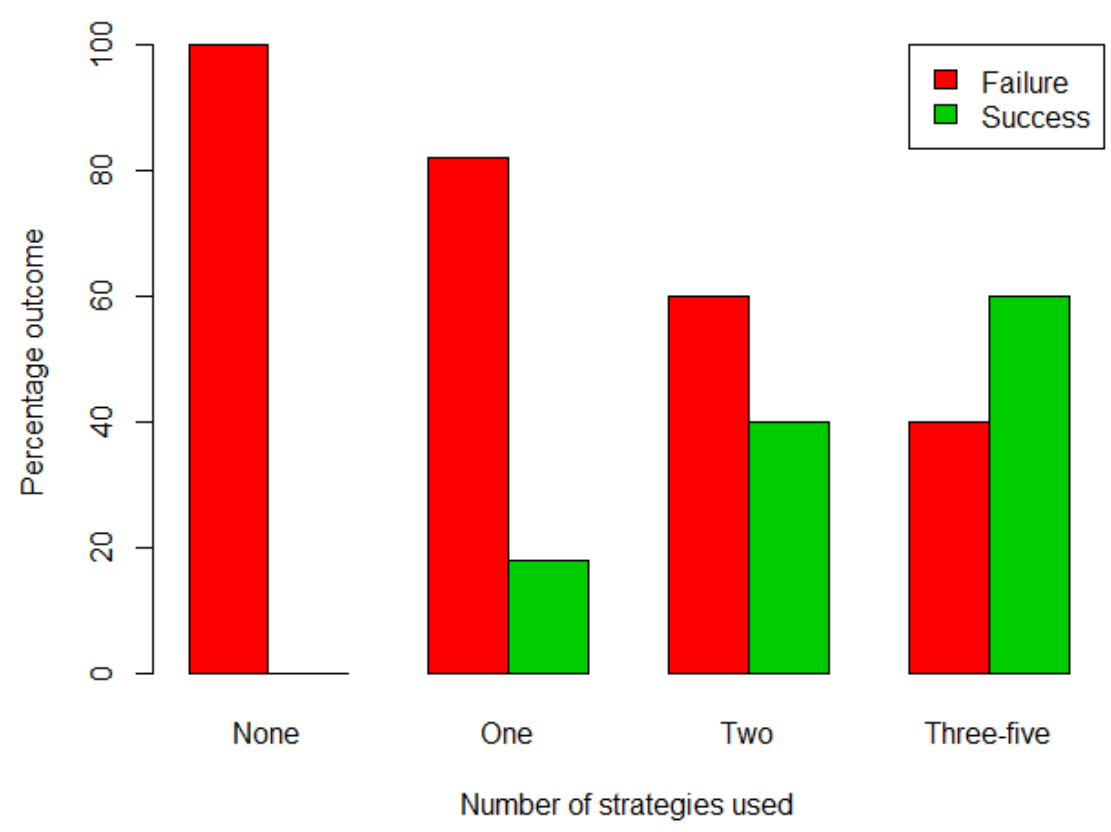

Figure 4: Percentage of success or failure as outcome of number of pre-emptive strategies for all steps taken together. 
This representation of the results may give a rough picture of the general tendencies, but it does not reveal the variation between steps or between participants and hence cannot be used as a basis on which to draw statistical conclusions. A more nuanced picture emerged when we looked at the steps individually. As can be seen in Table 4, the successful outcomes at each step included a higher average number of strategies than the unsuccessful outcomes (except for step 4, where we could not compare since there were no successful outcomes). ${ }^{5}$

Table 4: Mean number of pre-emptive strategies used for each outcome for each step of the instruction

\begin{tabular}{|l|l|l|}
\hline & Failure & Success \\
\hline Step 1 & 1.36 & 3.00 \\
\hline Step 2 & 1.86 & 2.80 \\
\hline Step 3 & 1.36 & 2.00 \\
\hline Step 4 & 0.92 & - \\
\hline Step 5 & 0.78 & 2.00 \\
\hline
\end{tabular}

In Figure 5, this tendency is illustrated in a different manner. The box diagrams demonstrate the mean number of pre-emptive strategies for each operator's failing and successful steps, respectively. In order to be able to carry out a pair-wise analysis, we included only conversations actually involving successful steps $(\mathrm{n}=13)$. Conversations lacking successful steps are hence also excluded from the following diagrams.

\footnotetext{
${ }^{5}$ It is worth noting about step 4 the low number of strategies employed and the total lack of successful outcomes. Also, as mentioned above, four participants skipped step 4 altogether. It seems reasonable to infer that step 4 has been perceived by some of the participants not as an autonomous step, but merely as preparation for step 5 . This could be due to the fact that step 4 does not entail any movement of the doll, in contrast with all the other steps. In addition, it may be the case that step 4 does not lend itself as easily to any of the pre-emptive strategies.
} 


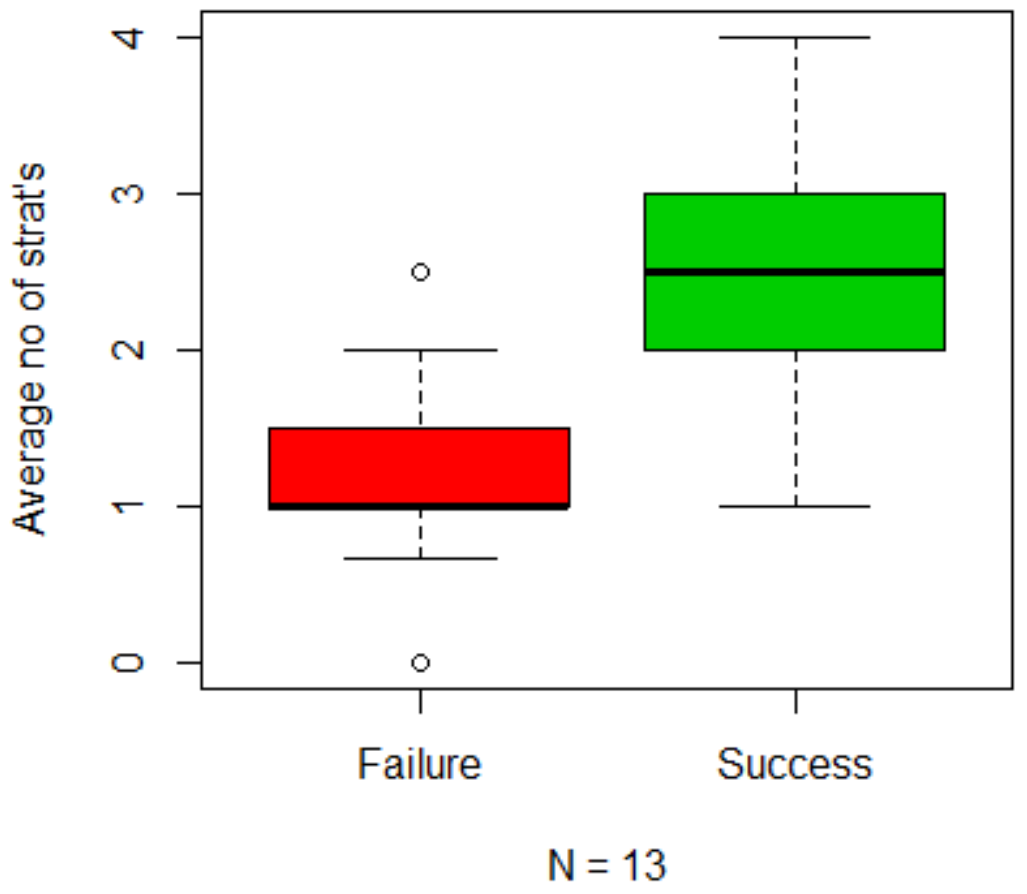

Figure 5: Mean number of pre-emptive strategies for each operator's failing and successful steps.

There was a certain degree of individual variation between the operators in that some used more pre-emptive strategies in general than did others. The individual differences between operators are demonstrated in Figure 6, which shows that all but two $(n=11)$ used more strategies when the outcome was successful than when it was unsuccessful (represented by a rising line). One operator had an equal number of strategies for successful and unsuccessful outcomes, and one had a lower number for successful than for unsuccessful outcomes. 


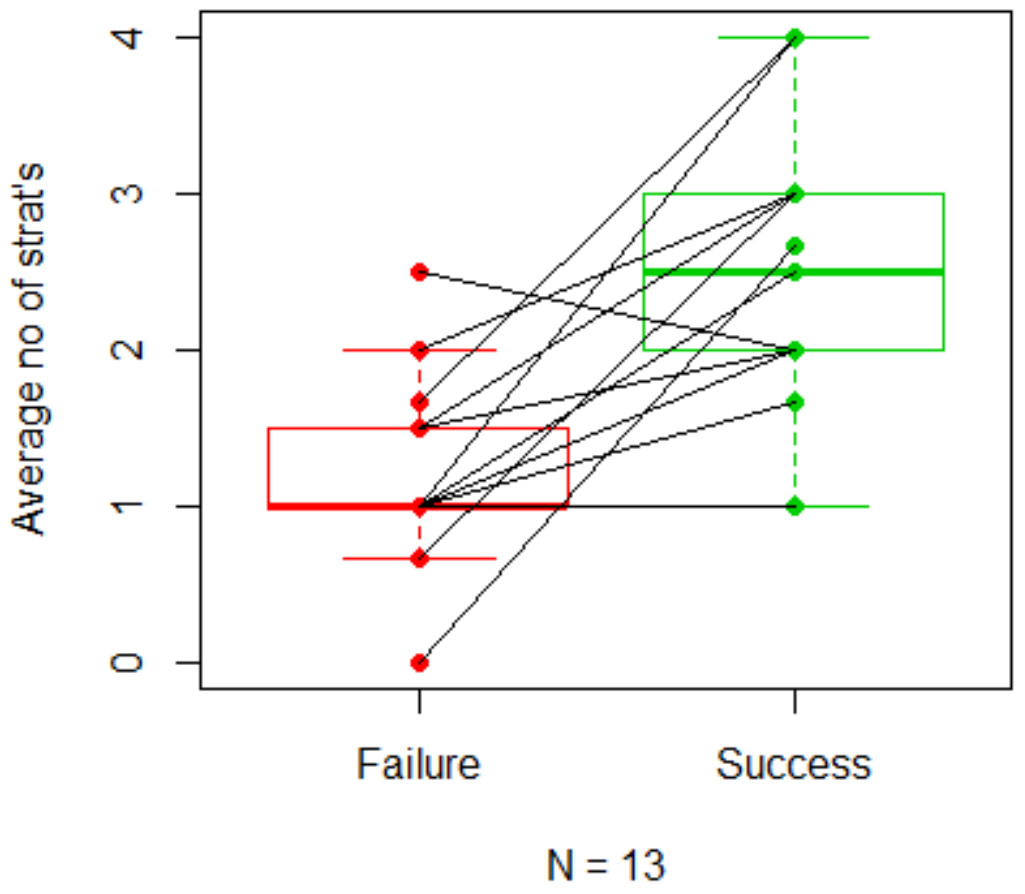

Figure 6: Individual profiles of operators.

Despite a rather low number of observations, the results are significant. We used a pairwise approach to the testing and employed a two-sided Wilcoxon signed rank test with continuity correction. The test resulted in a significant result, $\mathrm{V}=2, \mathrm{~N}=13, \mathrm{p}<0.01$, Cohen's $\mathrm{d} \approx 1.73$, i.e. it is likely that the tendency in the sample reflects a real difference in the population, and we can assume that a greater number of pre-emptive strategies employed in a step in general leads to a greater probability of success.

A possible objection to the validity of the results reported is a potential circularity in the definition of failure and the delimitation of the pre-emptive window. Since a display of nonunderstanding both closes the window and constitutes an instance of failure, it could be argued 
that the direction of the causality between the number of strategies employed and the rate of success is the opposite of the one we purport to investigate. Rather than a small number of strategies leading to a greater chance of failure, one could see the display of non-understanding, i.e. the failure, as closing the window, and hence precluding the employment of any further preemptive strategies for that step.

It is clear to us that such an objection is in part a valid one. In some instances, strategies are being employed successively (such as in excerpt (4), where several reformulations are added incrementally). In cases in which the caller does not understand, but stays silent, the operator may decide to employ further pre-emptive strategies, thus achieving understanding within the window, whereas in a similar situation in which the caller utters a signal of non-understanding, the window will be closed and the step associated with both failure and a smaller number of strategies. This would, however, in this case be due to the display of non-understanding rather than the operator's failure to employ any further strategies.

In many cases, however, several strategies occur together in the same utterance rather than sequenced in a succession of utterances (such as in excerpt (5), in which a left-dislocation, a reformulation and a simplification all occur within one and the same utterance). In order to partially validate the analysis, we carried out a supplementary analysis leaving out the strategy type most prone to be utilized in a successive manner, namely reformulation. The windows of investigation were left unaltered, but only simplification, decomposition and left-dislocation were counted as strategies. The supplementary analysis was carried out in the same manner as the original, i.e. with a paired Wilcoxon rank-sum test on the same sample of dialogues $(n=13)$, and it yielded a significant result, $\mathrm{W}=3, \mathrm{p}<0.01$, Cohen's $\mathrm{d} \approx 2.05$. This gives some support to our 
claim that the effect seen in the original analysis is not primarily the result of displays of nonunderstanding shortening the window of investigation.

Further, pre-emptive strategies should not be seen as an individual's choice, but as something that goes on in a co-operative interaction. A display of non-understanding often takes place at a natural point in the conversation, e.g. following a signal that the operator has come to an end in the explanation. Hence, we believe that the possible objection does not seriously affect the validity of the study. And finally, we do not see a way in which to study pre-emptive strategies in this kind of conversations without delimiting the window of investigation in the manner shown. To be able to investigate the phenomenon at all, the kind of compromise outlined in the above paragraphs seems to be necessary.

\section{Conclusion}

This study shows that L1 speakers intuitively work to prevent understanding problems by using pre-emptive strategies when giving instructions to L2 speakers with moderate proficiency in this context of emergency calls. They simplify potentially complicated lexical items, they reformulate (parts of) utterances that are potentially unclear, and they decompose complex information into smaller chunks (installments) and deliver them one at a time, monitoring for displays of understanding along the way. To a more limited extent, they also make new or important referents more salient by producing them in a separate intonation unit before the sentence frame (left-dislocation).

Such practices have been described in previous qualitative studies, but until now no researchers have attempted to investigate whether such pre-emptive practices actually lead to fewer understanding problems. The current quantitative analysis has shown that there is a 
relationship between the average number of strategies used and the chance of successful understanding; a greater number of strategies employed leads to fewer understanding problems.

The practical implications of this study are that intuitive strategies of preventing understanding problems are efficient and thus can be effective when speakers are unsure about whether or not they are being understood by their L2 interlocutors. Especially in cases where the speakers cannot see each other, such as emergency calls, verbal strategies are particularly important. Research has revealed that operators are less able to provide medical direction when speaking with L2 callers (Tate 2015); the present findings provide both suggestions for how to modify instructions for L2 callers and concrete evidence for their effectiveness. Previous research has also provided suggestions for how to simplify and rephrase potentially problematic instructions (Painter et al. 2014), and the present research shows that it may be most effective to use these strategies both in combination with each other and pre-emptively, rather than only as repair strategies once a problem of understanding has emerged. In other words, when speaking with L2 callers, emergency call operators (or other professionals) should be prepared to suspend progressivity in the service of making instructions more salient or accessible. These strategies contribute to a larger set of practices identified in recent analyses of emergency telephone calls with non-native speakers: Operators can systematize call openings with L2 callers to maximize efficiency (Penn et al. 2016) and assist callers in making their contributions as comprehensible as possible (Gerwing and Indseth 2016). In addition, when providing instructions, our research shows that emergency call operators and other professionals may be encouraged to use the four pre-emptive practices identified here and to use several of the strategies at once rather than just a single one. 
This study involves a special type of communicative activity, first aid instructions, and a special medium (telephone). The results are thus mainly relevant for this sort of interaction. Other activity types (explanations, information giving etc.) may involve other challenges and other types of strategies. And face-to-face interaction may provide other (visible) resources for establishing mutual understanding. To what extent the strategies investigated here are used also in other activity types and media is a question for further research.

Another limitation that needs to be pointed out, is that the study is not designed to explain the use of pre-emptive strategies by reference to the participants' language background as L1 and L2 speakers. We have not compared the conversations to similar instances involving only L1 speakers, so we cannot know to which degree the strategies are motivated by the L2 status of the caller rather than the communicative challenges involved in the task itself (and thus to which degree we would find the same strategies in L1/L1 conversations). Thus, systematic comparative studies are needed in order to assess the influence of the proficiency of L2 speakers on the use of pre-emptive strategies by L1 speakers in instructions (and possibly other types of communicative activities).

Finally, other remaining issues to be investigated in future studies include using actual emergency operators as a comparison group. Also experiments with operators before and after being trained in the use of pre-emptive strategies would yield interesting results in how much communication can be improved by initiatives oriented at raising operators' awareness.

\section{References}

Bavelas, J. B., J. Gerwing, S. Healing, \& C. Tomori. In press. 'Microanalysis of face-to-face dialogue: An inductive approach' in Van Lear, C. A. \& D. J. Canary (eds.): Researching 
Communication Interaction Behavior: A Sourcebook of Methods and Measures, p. 129157. Thousand Oaks, CA: SAGE.

Clark, H. H. 1996. Using Language. Cambridge: Cambridge University Press.

Clark, Herbert H. 1994. 'Managing problems in speaking,' Speech Communication 15: 243250.

Ferguson, C. A. 1975. 'Toward a characterization of English foreigner talk,' Anthropological Linguistics 17: 1-14.

Firth, A. \& J. Wagner. 1997. 'On discourse, communication, and (some) fundamental concepts in SLA research.' The Modern Language Journal 81: 285-300.

Gardner, R. 2004. 'On delaying the answer: Question sequences extended after the question' in Gardner, R. \& J. Wagner (eds.): Second Language Conversations, p. 246-266. London: Continuum.

Gerwing, J., \& T. Indseth. 2016. 'Enhancing comprehensibility in emergency calls with a language barrier: Operators in the role of addressee,' International Journal of Listening 30/3: 163-179. <http://dx.doi.org/10.1080/10904018.2015.1056878>

Grow, R. W., M. D. Sztajnkrycer, \& B. R. Moore. 2008. 'Language barriers as a reported cause of prehospital care delay in Minnesota,' Prehospital Emergency Care 12: 76-79.

Hatch, E. M. 1978. 'Discourse analysis and second language acquisition' in Hatch, E. (ed.): Second Language Acquisition: A Book of Readings, p. 401-435. Rowley, MA: Newbury House.

Heritage, J. 2007. 'Intersubjectivity and progressivity in person (and place) reference' in Enfield, Nick J. \& Tanya Stivers (eds.): Person Reference in Interaction, p. 255-80. Cambridge: Cambridge University Press.

Kasper, G. \& S. J. Ross. 2007. 'Multiple questions in oral proficiency interviews,' Journal of Pragmatics 40: 2045-2070.

Kendrick, K. 2017. 'Using Conversation Analysis in the lab.' Research on Language and Social Interaction 50: 1-11.

Lambrecht, K. 1994. Information Structure and Sentence Form: Topic, Focus, and the Mental Representation of Discourse Referents. Cambridge: Cambridge University Press.

Larsen, T. 2010. 'Jeg har brug for hjælp. Om anmodninger, alarmopkald og dansk som andetsprog' [I need help. On requests, emergency calls and Danish as a second language.] 
Nordand 5: 83-106.

Long, Michael H. 1996. 'The role of the linguistic environment in second language acquisition' in Ritchie W. C. \& T. K. Bhatia (ed.): Handbook of Second Language Acquisition, p. 413468. New York: Academic Press.

Mazeland, Harrie. 2007. 'Parenthetical sequences,' Journal of Pragmatics 39:1816-69.

Meischke, H., D. Chavez, S. M. Bradley, T. D. Rea \& M. Eisenberg. 2010. 'Emergency communications with limited-English-proficiency populations,' Prehospital Emergency Care 14: 1-7.

Meischke, H., B. Ike, I. Painter, D. Chavez, M. P. Yip, S. M. Bradley \& S. P. Tu. 2015. 'Delivering 9-1-1 CPR instructions to limited English proficient callers: a simulation experiment,' Journal of Immigrant and Minority Health 17: 1049-1054.

Osvaldsson, K., D. Persson-Thunqvist \& J. Cromdal, 2013. 'Comprehension checks, clarifications, and corrections in an emergency call with a nonnative speaker of Swedish', International Journal of Bilingualism, 17: 205-220.

Painter, I., D. E. Chavez, B. R. Ike, M. P. Yip, S. P. Tu, S. M. Bradley, T. D. Rea \& H. Meischke. 2014. 'Changes to DA-CPR instructions: Can we reduce time to first compression and improve quality of bystander CPR?,' Resuscitation 85: 1169-1173.

Penn, C., T. Koole \& R. Nattrass. 2016. 'When seconds count: A study of communication variables in the opening segment of emergency calls,' Journal of Health Psychology. DOI: $10.1177 / 1359105315625357$.

Pica, Teresa, Richard Young \& Catherine Doughty. 1987. 'The impact of interaction on comprehension,' TESOL Quarterly 21/4: 737-758.

Sacks, Harvey. 1984.' Notes on methodology' in Atkinson, Maxwell \& John Heritage (eds.): Structures of Social Action: Studies in Conversation Analysis. Cambridge: Cambridge University Press.

Sacks, Harvey, Emanuel Schegloff \& Gail Jefferson. 1974. 'A simplest systematics for the organization of turn-taking for conversation,' Language 50: 695-737.

Schegloff, Emanuel A., Gail Jefferson \& Harvey Sacks. 1977. 'The preference for selfcorrection in the organization of repair in conversation,' Language 53: 361-382.

Schiffrin, Deborah. 1987. Discourse Markers. Cambridge University Press, Cambridge.

Sidnell, Jack \& Tanya Stivers (eds.). 2013. The Handbook of Conversation Analysis. Oxford: 
Wiley-Blackwell.

Svennevig, Jan. 2010. 'Pre-empting reference problems in conversation,' Language in Society 39: 173-202.

Svennevig, Jan. 2012. 'On being heard in emergency calls. The development of hostility in a fatal emergency call,' Journal of Pragmatics 44/11: 1393-1412.

Svennevig, Jan. 2013. 'Reformulation of questions with candidate answers,' International Journal of Bilingualism 17/2: 189-204.

Svennevig, Jan. 2015. 'Én porsjon om gangen. Multimodal koordinering i samtaler med andrespråksbrukere' [One portion at a time. Multimodal coordination in conversation with L2 users] i Engebretsen, M. (red.): Det tredje språket. Multimodale studier av interkulturell kommunikasjon i kunst, skole og samfunnsliv [The third language. Multimodal studies of intercultural communication in art, school and society], p. 196-211. Kristiansand: Portal forlag.

Tate, R. C. 2015. 'The need for more prehospital research on language barriers: A narrative review,' Western Journal of Emergency Medicine 16: 1094-1104.

Varonis, E. M. \& Susan M. Gass. 1985. 'Native/non-native conversations: A model for negotiation of meaning,' Applied Linguistics 6/1: 71-90.

Wittenburg, P., H. Brugman, A. Russel, A. Klassmann \& H. Sloetjes. 2006. 'ELAN: A Professional Framework for Multimodality Research' in Proceedings of LREC 2006, Fifth International Conference on Language Resources and Evaluation, p. 1556-1559. 